\title{
Saliva Crystallization in Sheep Subjected to Estrus Induction and Synchronization Protocols
}

\author{
Andressa Silveira Gonçalves, Eneder Rosana Oberst \& Raquel Fraga e Silva Raimondo
}

\begin{abstract}
Background: The crystallization of bodily fluids, primarily saliva, has been the subject of study in many species and is a simple alternative to detect estrus because it demands neither a significant financial investment nor qualified professionals to execute the examination. Fern pattern crystallization has been described in the cervical and nasal mucus, saliva and tear secretion, and in colostrum. Changes in salivary crystallization during the reproduction cycle are related to different hormonal concentrations in this period. Thus, the present study has evaluated the patterns of saliva crystallization in sheep subjected to estrus induction and synchronization protocols.

Materials, Methods \& Results: The sample consisted of 11 crossbreed Corriedale sheep, which were evaluated during two experimental periods (spring and autumn), and that underwent induction and synchronizing estrus protocols. In a random phase of the estrus cycle (day 0), each sheep was implanted with an intravaginal device (Primer®), impregnated with 0.36 $\mathrm{g}$ of progesterone for seven days. This device was inserted according to manufacturer's instructions of the manufacturer and with the assistance of a specific applicator. On the day of device removal (day 7), the animals received $0.0375 \mathrm{mg}$ of D-Cloprostenol (Prolise ${ }^{\circledR}$ ) and $10 \mathrm{mg}$ of Folltropin ${ }^{\circledR}$ extracted from the swine pituitary (NIH-FSH-P1 of Folltropin-V) by intramuscular administration. The saliva was collected at six points during the experimental periods: day 1 ( 3 days before placement of the implant); day 4 (day of insertion of the implant); day 9 ( 5 days after the insertion of the implant); day 11 (day of removal of the implant and application of hormones); day 12 (24 h after removal of the implant [presumable estrus]); and day 13 ( $48 \mathrm{~h}$ after removal of the implant). Smears containing $10 \mu \mathrm{L}$ of saliva were observed under an optical light microscope $(\mathrm{x} 200)$ for assessment of saliva crystallization. Salivary crystallization was classified in scores from 0 to 3 based on the extent of crystallization observed in the slide, also considering filling of the observation field (x200) and type of arborization pattern observed, as follows: $0=$ without crystallization; $1=$ too little crystallization; $2=$ low crystallization; and 3= large amount of crystallization. The patterns of crystallization found in sheep demonstrated an increase in quantity, diversity, definition, and size of sheets as the period of estrus approached and were different than those tipically described in the literature. Patterns of salivary crystallization followed the phases of the estrus cycle defined by the estrus induction and synchronization protocol.

Discussion: Salivary crystallization in the sheep used in this study exhibited patterns that were different than those typically described in the literature. In the spring, salivary crystallization score demonstrated variation from day 1 to 11 of the protocol, when the predominant hormone was progesterone released by the implant (diestrus). However, in Autumn, on day 4 (implant placement day), an increase in crystallization score was noted. This may have occurred because the sheep were in the reproductive season and therefore, may have been nearing seasonal estrus before placement of the implant. The patterns of crystallization found in sheep demonstrated an increase in quantity, diversity, definition, and size of sheets as the period of estrus approaches and showed patterns that were different than the ones usually described in literature. The patterns of salivary crystallization followed the phases of the estrus cycle defined by the estrus induction and synchronization protocol.
\end{abstract}

Keywords: ewe, estrus, reproduction, ovine. 


\section{INTRODUCTION}

In the ovine, seasonality is related to a negative photoperiod and the breeding season normally begins at the end of summer and the beginning of autumn, in response to a decrease in luminosity. The anestrus period comprises both the end of winter and the beginning of spring. This situation has a direct effect on the birth of lambs, restricting it to a determined period of the year. Aside from seasonality another obstacle is the span of estrus, which has an average duration of $38 \mathrm{~h}$, with the signs exhibited by sheep being less evident than in other ruminant species.

Analysis of crystallization patterns influenced by the presence of estrogen in bodily fluids, primarily in the cervical mucus, has been used to monitor the reproductive cycle for more than 60 years in the human species. Crystallization can be defined as the process by which a component of a liquid solution changes into its solid phase, tending to separate from the solution and precipitate in the form of crystals [9]. In the bovine, cervical mucus exhibits variations over the entire cycle, with the presence of the fern pattern during estrus and its absence in other phases of the cycle [2]. Crystalline forms have been termed "arborization" or the "fern phenomenom" [3]. The use of saliva to evaluate patterns of crystallization related to the estrus cycle is considered to be a less invasive procedure compared with the collection of cervical mucus. Changes in salivary crystallization during the reproduction cycle are related to different hormonal concentrations in this period, mainly estrogen, which is the hormone responsible for the elevation of electrolytes, levels forming the crystals [3]. The objective the present study was to evaluate the patterns of saliva crystallization in sheep subjected to estrus induction and synchronization protocols.

\section{MATERIALS AND METHODS}

\section{Experimental design}

The present study was conducted in the ovine breeding facility of Faculty of Veterinary Medicine, Universidade Federal do Rio Grande do Sul (UFRGS), located in the city of Porto Alegre, RS, in the southern region of Brazil $\left(30^{\circ} 01^{\prime} 40^{\prime \prime} \mathrm{S}, 51^{\circ} 13^{\prime} 43^{\prime \prime} \mathrm{W}\right)$. A total of 11 multiparous, 2-6-years-old crossbred Corriedale sheep were experimentally evaluated on two periods: spring (November 1 to November 13, 2016) and autumn (March 31 to April 12, 2017). Only dry healthy ewes with a body condition score between 3 and 4 in a 5-point scale ( $1=$ emaciated, $5=$ obese) [6] at the beginning of synchronization treatment, were included in the study. The animals were housed in a paddock with improved native pasture, containing cultivated millet (Pennisetum americanum) and aruana grass (Panicum Maximum) and ad libitum access to water.

Seeking to equalize the phases of the estrus cycle and estimate the day of estrus, the sheep were subjected to short estrus induction and synchronization protocols, which were implemented both out of the breeding season (spring) and during the breeding season (autumn). In a random phase of the estrus cycle (day 0), each sheep was implanted with an intravaginal device $\left(\text { Primer }^{\circledR}\right)^{1}$, impregnated with 0.36 $\mathrm{g}$ of progesterone for seven days. This device was inserted according to manufacturer's instructions of the manufacturer and with the assistance of a specific applicator. On the day of device removal (day 7), the animals received $0.0375 \mathrm{mg}$ of D-Cloprostenol ${ }^{1}$ (Prolise ${ }^{\circledR}$ ) and $10 \mathrm{mg}$ of Folltropin ${ }^{1}$ extracted from the swine pituitary (NIH-FSH-P1 of Folltropin) by intramuscular administration.

\section{Saliva collection and crystallization}

The collection of saliva was performed at six points during the experimental periods: day 1 ( 3 days before placement of the implant); day 4 (day of insertion of the implant); day 9 ( 5 days after the insertion of the implant); day 11 (day of removal of the implant and application of hormones); day 12 (24 h after removal of the implant [presumable estrus]); and day 13 (48 h after removal of the implant) [Figure 1].

The sheep were restrained and a gauze bandage prevented mouth opening as well as promoted the pooling of saliva, which was collected using a disposable Pasteur pipette inserted in the labial commissure. The collection of saliva was performed daily at approximately 13:30 h. The collected material was stored in microtubes under refrigeration until decantation, (a process that took approximately $1 \mathrm{~h}$, to remove any particles of food, dirt, or cells. A $10 \mu \mathrm{L}$ fraction of the saliva supernatant was used to prepare a smear with circular movements of the drop applied to a glass slide. Subsequently, the smears were identified, dried at $39^{\circ} \mathrm{C}$ on a thermic table for fixation, for approximately 15 min and, later, observed under light microscopy (CX 
40)2 , Olympus, under 200 magnification for the classification of salivary crystallization, using a standard adapted from the study by Ravinder et al. [5]. Salivary crystallization was classified in scores from 0 to 3 based on the extent of crystallization observed in the slide, also considering filling of the observation field (x200) and type of arborization pattern observed, as follows: $0=$ without crystallization; 1 = too little crystallization; 2 = low crystallization; and $3=$ large amount of crystallization.

\section{Statistical analysis}

The salivary crystallization scores were submitted to analysis of variance for repeated measures by generalized linear models (GLM / GEE) SPSS $^{3}$ version 18 (IBM Corporation, Armonk, NY, USA) andexpressed as mean and standard error of the mean. The means were compared taking into consideration the days over each experimental period and comparing the day in each experimental period (autumn x spring) through the Bonferroni test, $P<0.05$ was considered to be statistically significant.

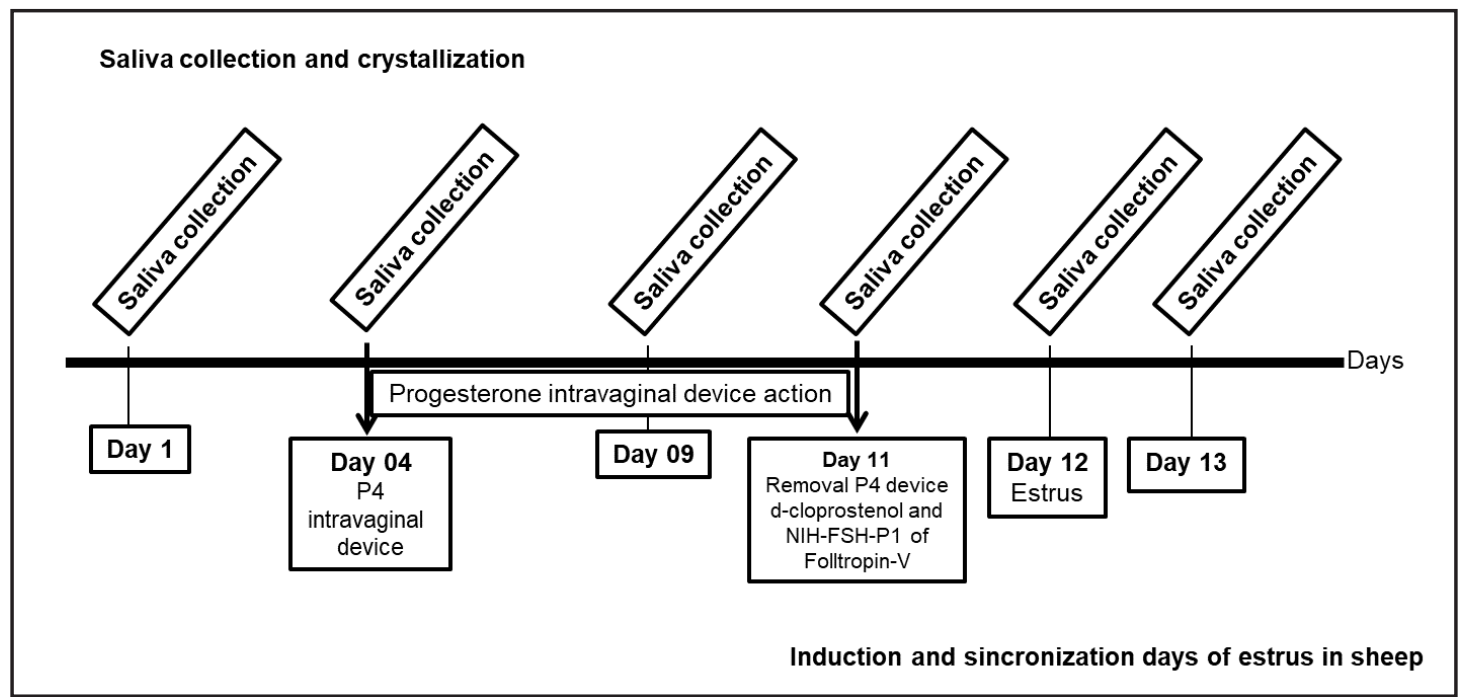

Figure 1. Time line of the sample collections during days of estrus induction and synchronization protocols during the two experimental periods, spring and autumn, to evaluate the pattern of salivary crystallization in sheep.

\section{RESULTS}

Salivary crystallization in the sheep used in this study exhibited patterns that were different than those typically described in the literature.

Designs similar to "snowflakes" (Figure 2A) were observed, and characterized by small leaves without branches or stems, which are observed more in metaestrus. Larger, more robust and rounded leaf-shaped crystals (Figure 2B) were observed on the blades during prestrus and estrus. Differentiated, rounded and welldelimited formats similar to the Amazon plant "victor regia" (Figure 2C) were found in some blades during the estrus period (day 12). Some crystallizations were rounded, but irregular and without definite delimitation, appearing to be arranged one above the other, comparable to "fungal colonies" (Figure 2D) and were exhibited near estrus. Elongated leaves similar to branches, with long and well delimited stems were observed only during estrus. In some cases, several patterns of different designs were observed on the same blade. Another interesting aspect noted was that most of the slides exhibited more than one type of crystallization and some sheep exhibited unique crystallization patterns, as all the slides of a given animal contained the same drawings, varying only their amount and distribution on the slide.

Because the crystallization patterns observed in the present study did not resemble the afforestation patterns described in the literature, the authors decided to use a scoring classification from 0 to 3 , based on the extent of crystallization observed on the slides, considering the filling of the observation field (x200) and the type of afforestation observed. The scoring system was as follows: " 0 = no crystallization" (Figure 3 ), characterized by very few and very small misshapen crystals, almost as crystallization dots with little filling of the slide; "1 = very little crystallization" (Figure 3 ) characterized by an observation range from small crystals with snowflake 
shapes to slightly larger leaves with little blade filling; " 2 $=$ little crystallization" (Figure 4) larger, more robust and rounded leaves appear, which may present forms alike "victor regia" leaves and "fungal colonies", and few uncrystallized spaces on the blade; " $3=$ large amount of crystallization" (Figure 4) all other previous forms were observed, such as "snowflakes", more robust and rounded leaves, "Victor regia" forms, "fungal colony" forms and elongated leaves, with virtually the entire blade filled with crystallization.

The patterns of salivary crystallization observed in the ewes followed the phases of the estrus cycle, defined by the estrus induction and synchronization protocol used. During autumn (Figure 5 and Table 1), the lowest score of salivary crystallization was observed on day 1 of the protocol, and the highest was observed on day 12
(24 $\mathrm{h}$ after removal of the progesterone implant and hormone application). Subsequently, there was a significant decrease in the crystallization score at day 13 . We also observed an increase in salivary crystallization score on day 4 of the protocol (day of implant placement) that remained stable through days 9 and 11 was also observed.

In the spring (Figure 5 and Table 1) salivary crystallization score demonstrated no variation from days 1 to 11 , reaching to their maximum values on day 12 , with no difference between days. Similar to what was observed in autumn, there was a significant decrease in crystallization score on day 13 compared with day 12 . The higher crystallization score was also noted on day12, although without significant variation. A significant seasonal difference in crystallization scores was observed in autumn and spring, on days 4 and 12.

Table 1. Mean, standard deviation and confidence interval of the salivary crystallization scores of sheep obtained during the days of estrus induction and synchronization protocols during the two experimental periods, spring and autumn.

\begin{tabular}{|c|c|c|c|c|c|c|c|c|}
\hline \multirow{3}{*}{ Day } & \multicolumn{4}{|c|}{ Autumm } & \multicolumn{4}{|c|}{ Spring } \\
\hline & \multirow{2}{*}{ Mean } & \multirow{2}{*}{ SD } & \multicolumn{2}{|c|}{ 95\% Wald Confidence Interval } & \multirow{2}{*}{ Mean } & \multirow{2}{*}{$\mathrm{SD}$} & \multicolumn{2}{|c|}{ 95\% Wald Confidence Interval } \\
\hline & & & Lower & Upper & & & Lower & Upper \\
\hline Day 01 & $1.27^{\mathrm{a}}$ & 0.34 & 0.60 & 1.94 & $0.82^{\mathrm{ab}}$ & 0.31 & 0.21 & 1.43 \\
\hline Day 04 & $2.64^{\mathrm{bc}}$ & 0.15 & 2.35 & 2.92 & $1.55^{\mathrm{ab}}$ & 0.27 & 1.02 & 2.07 \\
\hline Day 09 & $1.45^{\mathrm{a}}$ & 0.30 & 0.87 & 2.04 & $1.55^{\mathrm{ab}}$ & 0.30 & 0.96 & 2.13 \\
\hline Day 11 & $1.45^{\mathrm{a}}$ & 0.32 & 0.82 & 2.09 & $1.55^{\mathrm{ab}}$ & 0.35 & 0.86 & 2.23 \\
\hline Day 12 & $2.82^{\mathrm{b}}$ & 0.12 & 2.59 & 3.05 & $1.82^{\mathrm{a}}$ & 0.31 & 1.21 & 2.43 \\
\hline Day 13 & $1.55^{\mathrm{ac}}$ & 0.43 & 0.70 & 2.39 & $0.55^{\mathrm{b}}$ & 0.20 & 0.16 & 0.93 \\
\hline
\end{tabular}

Different letters in the column indicate a statistical difference at $P<0.05$ by Bonferroni test.

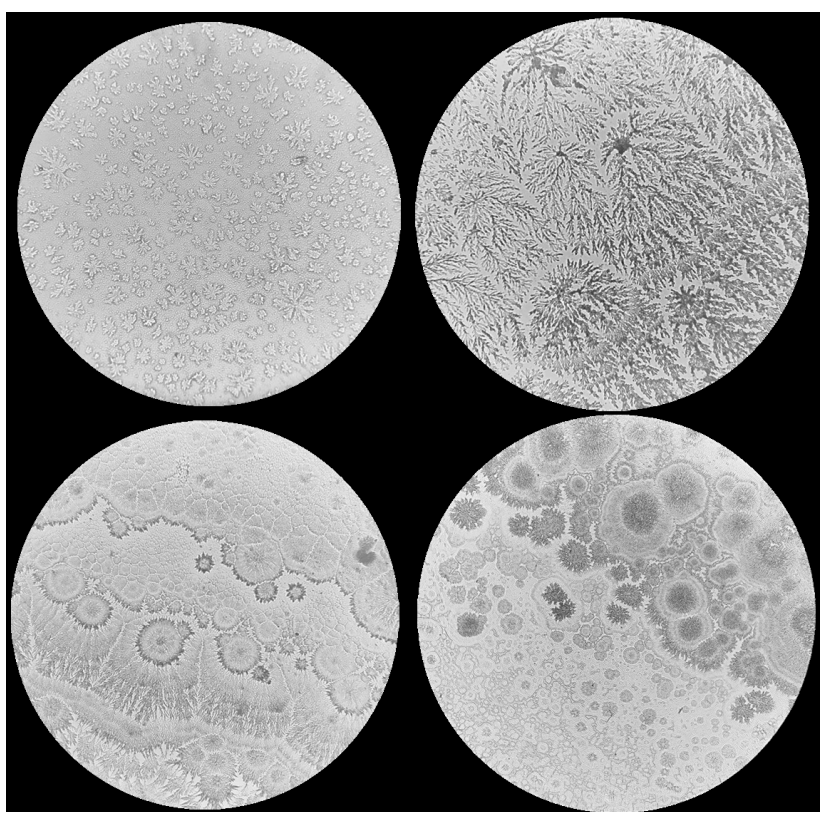

Figure 2. Different salivary crystallization patterns in sheep submitted to estrus induction and synchronization protocols. Microscopic images of dried saliva [200x]. A- Snowflakes. B- Crystals in the form of larger, more robust, and rounded leaves. C- Victor-regia plant. D- Fungal colonies.

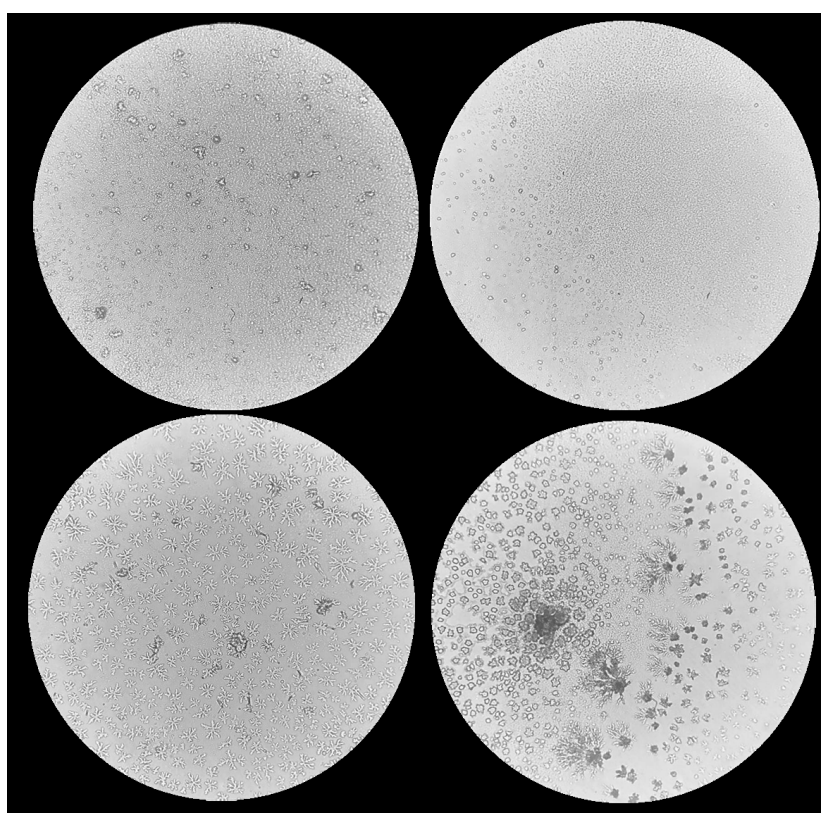

Figure 3. Different salivary crystallization patterns in sheep submitted to estrus induction and synchronization protocols. Microscopic images of dried saliva [200x]. Scoring Classification: 0- no crystallization and 1 - very little crystallization. 


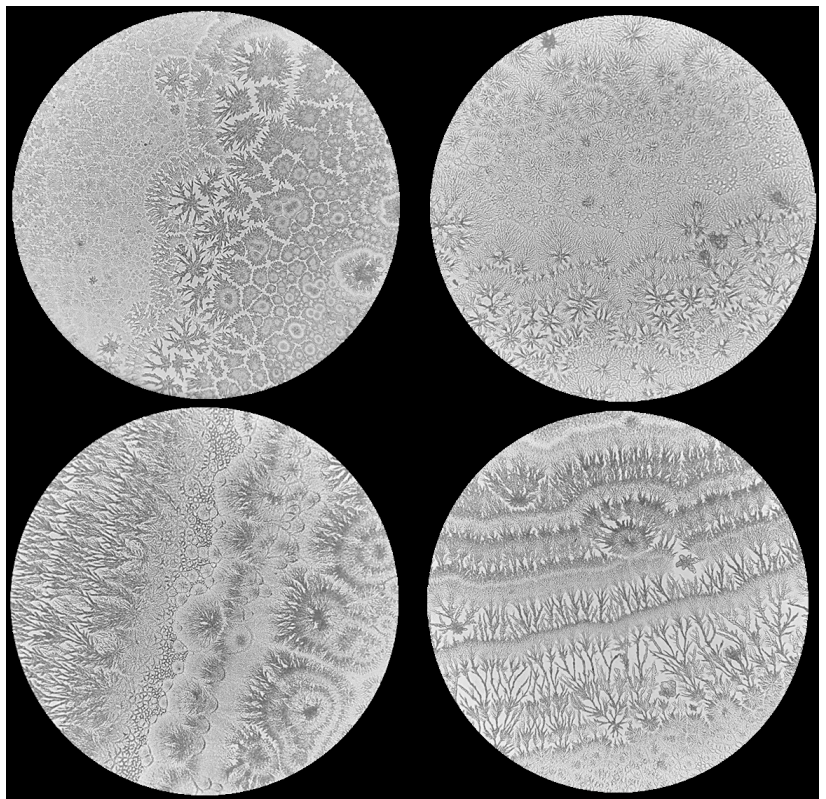

Figure 4. Different salivary crystallization patterns in sheep submitted to estrus induction and synchronization protocols [200x]. Scoring Classification: 2- little crystallization and 3- large amount of crystallization.

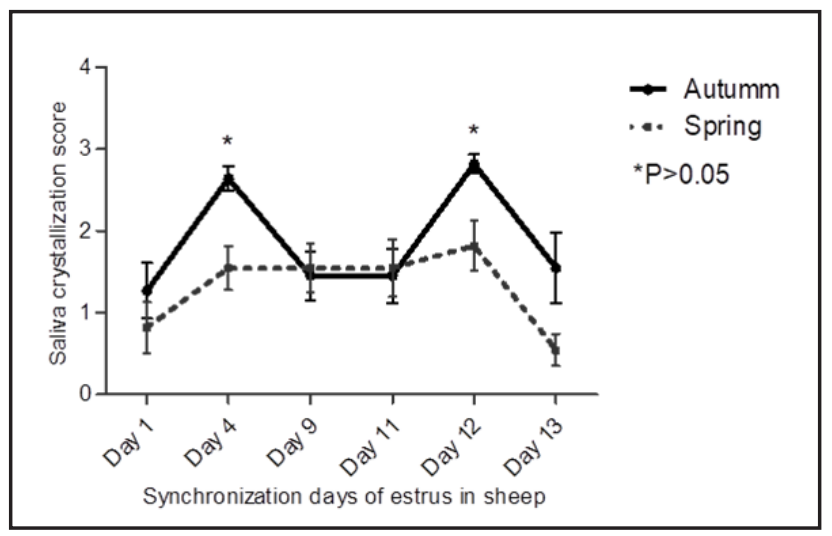

Figure 5. Comparison of the salivary crystallization score of sheep during reproductive season (autumn) and out of reproductive season (spring) during the days of estrus hormonal synchronization. *Indicate a statistical difference at $P<0.05$ by Bonferroni test in day between seasons.

\section{DISCUSSION}

We classified sheep salivary crystallization according to scores from 0 (without crystallization) up to 3 (large amount of crystallization) based on the extent of crystallization observed on the slide, while also considering filling of the observation field (x 200) and type of arborization pattern observed. In recent studies evaluating salivary crystallization among different phases of the estrus cycle, several classifications of crystallization patterns were adopted based on afforestation profile. Two ratings were used in orangutans. In the first, the authors chose the classification used for humans, which in non-fertile days exhibited neither crystallization nor small random crystals on the slide. During the transition period crystallization patterns exhibited intermediate crystals combined with dots and other forms of crystallization including branch and, fir-like, and fern-like type, which was also a pattern tipically observed in the fertile period in the fertile period [3]. Regarding the second classification, which is most commonly used for animals, the method used by the authors was the same as described in bovine [8] which used the following scale: without crystallization (none), branch-like, fir-like, fern-like, and its combinations (branch-like and fir-like, branch-like and fern-like and branch-like, fir-like, and fern-like).

In buffaloes, six different crystallization patterns were observed: the typical symmetrical fern-like pattern, branch-like, fir-like, combinations of fir-fernbranch types, dotted, and none [5]. In sheep, there are very few studies on salivary crystallization during the estrus cycle or in estrus synchronization protocols. In a study examining sheep during the seasonal estrus cycle, short-stem fern patterns were observed during prestrus; throught estrus, clear crystallization patterns were found with long and clear stems; over metaestrus the samples exhibited scattered irregular patterns with small ferns, and during diestrus, there was no evidence of crystallization [7]. The authors observed no uniform fern-like pattern, however, there were different characteristics in the ferns linked to the stage of the estrus cycle. This result may be a consequence of the chosen hormonal treatment, which may have altered typical characteristics of the salivary crystallization pattern expected to occur in natural estrus.

The increase in salivary crystallization intensity, presented as scores, according to our method, was a good indicator for the determination of estrus in sheep, because the patterns of salivary crystallization observed in the ewes followed the phases of the estrus cycle, defined by the estrus induction and synchronization protocol that was used.

In the autumn and spring, the highest salivary crystallization score was recorded on day 12 of the protocol, (24 h after removal of the progesterone implant and hormone application), the day of possible estrus and higher estrogen concentration, according to the protocol used. Subsequently, there was a significant decrease in crystallization score at day 13. Changes in saliva secretion, and nasal and cervical mucus, associated with crystalliza- 
tion are related to the secretion of estrogen and progesterone, however, while estrogen favors crystallization, progesterone inhibits it [2-4,7]. Thereafter, the highest salivary crystallization score was noted on day 12 , with a significant decrease in score at day 13 , which suggests that day 12 may have been the actual estrus day, when a higher crystallization score is expected.

In the spring, salivary crystallization score demonstrated variation from day 1 to 11 of the protocol, when the predominant hormone was progesterone released by the implant (diestrus). However, in Autumn, on day 4 (implant placement day), an increase in crystallization score was noted. This may have occurred because the sheep were in the reproductive season and therefore, may have been nearing seasonal estrus before placement of the implant.

When we compared days of collection between the two experimental periods, (i.e., autumn, and spring), we observed that crystallization scores on days 4 and 12 in the autumn were significantly higher than those observed in the spring. The significant difference on day 4 may have occurred because all crystallization scores observed in autumn were higher than those in spring. Furthermore, the sheep may have started cyclic activity during autumn.

On day 12, the day of possible estrus and higher estrogen concentration, according to the protocol used, the saliva crystallization score of sheep during the reproductive season was significantly higher compared with the spring scores. The lower salivary crystallization observed during spring and the greater crystallization observed on estrus day in autumn could be caused by environmental conditions, especially due to the action of a decreasing photoperiod during autumn, which is the physiological period of better reproductive performance.

Therefore, in autumn, the modifications determined by synchronization were positively enhanced by the favorable environment, thus increasing the intensity of the crystallization pattern. It is worth noting that even with the use of stimulatory hormones, follicular growth is more expressive in the reproductive season than during the anestrus period [1].
The fact that hormonal dosages were not performed is a limitation of the present study, since the results would clearly indicate the day of estrus. However, estrus synchronization allows us to know the days of the hormonal changes and the day of possible estrus. Thus, the observed differences in salivary crystallization over the days and according to the time of the year in which the synchronization protocol was performed, probably reflect the hormonal response of the sheep to the protocols used.

\section{CONCLUSIONS}

The patterns of crystallization found in sheep demonstrated an increase in quantity, diversity, definition, and size of sheets as the period of estrus approaches and showed patterns that were different than the ones usually described in literature. The patterns of salivary crystallization followed the phases of the estrus cycle defined by the estrus induction and synchronization protocol. On day 12 (date of possible estrus and higher estrogen concentration, according to the protocol used) the saliva crystallization score of sheep during reproductive season was significantly higher when compared to the spring scores. Future studies evaluating the use of salivary crystallization in natural and large-scale estrus are timely.

\section{MANUFACTURERS}

${ }^{1}$ Tecnopec Linha Reprodutiva. Agener União, São Paulo, SP, Brazil. ${ }^{2}$ Olympus Corporation. Tokyo, Japan.

${ }^{3}$ IBM Corporation. Armonk, NY, USA.

Acknowledgments. Agener União Saúde Animal and members of Núcleo RuminAção, ensino, pesquisa e extensão em ruminantes, Faculdade de Veterinária, Universidade Federal do Rio Grande do Sul (UFRGS).

Ethical approval. All procedures care was in compliance with the Ethics Committee on Animal Experimentation of the Federal University of Rio Grande do Sul, and the research was registered and approved under the number 32772 .

Declaration of interest. The authors report no conflicts of interest. The authors alone are responsible for the content and writing of the paper.

\section{REFERENCES}

1 Barrett D.M.W., Bartlewski P.M., Duggavathi R., Davies K.L., Huchkowsky S.L., Epp T. \& Rawlings N.C. 2008. Synchronization of follicular wave emergence in the seasonally anestrous ewe: The effects of estradiol with or without medroxyprogesterone acetate. Theriogenology 69(7): 827-836.

2 Gnanamuthu G. \& Rameshkumar K. 2015. Determination of estrus in umblachery cattle (Bos indicus) by salivary fern pattern. International Journal for Species. 13(41): 68-78. 
3 Kubátová A. \& Fedorova T. 2016. Saliva crystallization occurs in female bornean orangutans (Pongo pygmaeus): Could it be a new option for monitoring of menstrual cycle in captive great apes? PLoS One. 11(7): 1-11.

4 Pardo-Carmona B., Moyano M.R., Fernández-Palacios R. \& Pérez-Marín C.C. 2010. Saliva crystallisation as a means of determining optimal mating time in bitches. Journal of Small Animal Practice. 51(5): 437-442.

5 Ravinder R., Kaipa O., Baddela V.S., Singhal Sinha E., Singh P., Nayan V., Velagala C.S.N., Baithalu R.K., Onteru S.K. \& Singh D. 2016. Saliva ferning, an unorthodox estrus detection method in water buffaloes (Bubalus bubalis). Theriogenology 86(5): 1147-1155.

6 Russel A.J.F., Doney J.M. \& Gunn R.G. 1969. Subjective assessment of body fat in live sheep. Journal of Agricultural Science. 72(3): 451-454.

7 Sangeetha P. \& Rameshkumar K. 2015. Detection of Estrus in Sheep (Ovis aries) by Salivary Fern Pattern and Vaginal Cytological Examination with Relation to Estrogen. The Scitech Journal. 2(5): 29-33.

8 Skalova I., Fedorova T. \& Brandlova K. 2013. Saliva Crystallization in Cattle: New Possibility for Early Pregnancy Diagnosis? Agricultura Tropica et Subtropica. 46(3): 102-104.

9 Weber P.C. 1991. Physical Principles of Protein Crystallization. Advances in Protein Chemistry. 41: 1-36. 